\title{
Head Position of Patients with Right Hemisphere Damage during a Visual Search Task in a Large Field
}

\section{Ken Nakatani ${ }^{1}$, Shusuke Takahashi' ${ }^{2}$, Daisuke Kimura1, Masako Notoya ${ }^{3}$, Nobuyuki Sunahara ${ }^{4}$, Takashi Fujita $^{5}$, Minoru Toyama ${ }^{3}$, Shinya Fukunaga ${ }^{6}$, Naoe Mori'1, Kiyoe Sakai' Shinya Fuwa ${ }^{1}$}

\author{
${ }^{1}$ Department of Rehabilitation Sciences, Kansai University of Welfare Sciences, Osaka, Japan \\ ${ }^{2}$ Department of Living and Welfare, Faculty of Home Economics, Kyoto Women's University, Kyoto, Japan \\ ${ }^{3}$ Department of Speech and Hearing Sciences and Disorders, Kyoto Gakuen University, Kyoto, Japan \\ ${ }^{4}$ School of Health Sciences, College of Medical, Pharmaceutical, and Health Sciences, Kanazawa University, Kanazawa, Japan \\ ${ }^{5}$ Division of Occupational Therapy, Faculty of Care and Rehabilitation, Seijoh University, Tōkai, Japan \\ ${ }^{6}$ Department of Sensory Science, Faculty of Health Science and Technology, Kawasaki University of Medical Welfare, Kurashiki, \\ Japan \\ Email:knaka62735@gmail.com
}

How to cite this paper: Nakatani, K. Takahashi, S., Kimura, D., Notoya, M. Sunahara, N., Fujita, T., Toyama, M., Fukunaga, S., Mori, N., Sakai, K. and Fuwa, S. (2018) Head Position of Patients with Right Hemisphere Damage during a Visual Search Task in a Large Field. World Journal of Neuroscience, 8, 190-202. https://doi.org/10.4236/wjns.2018.82018

Received: March 6, 2018

Accepted: May 8, 2018

Published: May 11, 2018

Copyright (C) 2018 by authors and Scientific Research Publishing Inc. This work is licensed under the Creative Commons Attribution International License (CC BY 4.0).

http://creativecommons.org/licenses/by/4.0/

\begin{abstract}
Spatial neglect syndrome with cerebral lesions is characterized by the inability to orient, report, or respond to relevant visual stimuli contralateral to the lesions. In this study, we investigated the pattern of horizontal visual search in a large space; this search was performed by patients with right hemisphere damage caused by cerebrovascular disease. The neck rotation angle and search time in each increment were continuously recorded during the task, and quantitative data of the measurements were collected. Head position during the visual search task in a large space (\%) was then calculated. We set angular bands in increments of 5 degrees from the midline on the left and right sides, and calculated the total search time in each angular band. In patients with unilateral spatial neglect (USN), the search time in the angular band of 5 - 9 on the right side was significantly longer (with USN 16.7\%, without USN 4.5\%, $t$ $=2.52, \mathrm{df}=16, \mathrm{p}<0.05)$. Furthermore, in patients with neglect in the leftmost end area in a large space, the search time was significantly shorter in the angular band of $1-4$ degrees to the left (with neglect 13.1\%, without neglect $23.7 \%, t=2.13, \mathrm{df}=16, \mathrm{p}<0.05$ ), and tended to be long in the angular band of 5 - 9 to the right. The neck rotation angle and pattern during the search task in a large field deviated slightly to the right, and the search time slightly to the left was short. We believe that these results support the rightward deviation of the search pattern and frame of USN patients.
\end{abstract}




\section{Keywords}

Head Position, Neck Rotation Angle, Unilateral Spatial Neglect, Visual Exploration

\section{Introduction}

Spatial neglect syndrome in patients with a cerebral lesion is defined as the failure to orient, report, or respond to relevant visual stimuli contralateral to the lesion [1]. The incidence of unilateral spatial neglect (USN) is high in patients with right hemisphere damage, and USN can be an impediment to rehabilitation. In clinical practice, a desk-based test in a small space is frequently performed to evaluate the severity of USN, and the deviation in the horizontal direction (horizontal axis), mainly left-side USN, is considered. In recent years, several studies have investigated the diversity of symptoms of spatial neglect, including vertical neglect [2] [3] and peripersonal/extrapersonal neglect [3] [4] [5]. We have previously reported on the diversity of USN symptoms, wherein we focused on a sound lateralization ability using auditory tasks [6], as well as on a visual search ability and horizontal neck rotation angle during visual search tasks in a large space [7]. In our previous study, we divided subjects into two groups, one with USN and one without USN, based on the score of Behavioural Inattention Test Conventional Subtest (BITC). By analyzing horizontal visual search ability, head position, and neck rotation pattern during the search task in a large space, we reported a strong correlation between the number of visual search tasks performed in a large space and the total BITC score. Furthermore, no significant difference was observed with respect to the neck rotation angle as well as the average and maximum values of the two groups [7].

However, it remains unclear as to what types of neck rotation patterns the lower score group shows and what types of neck rotation patterns the group that neglects the leftmost end area in a large space, after dividing the subjects based on the scores of the search task in a large space. Few studies have quantified the search pattern of USN patients by continuously measuring the neck rotation angle with respect to the tasks continuously presented in a large space. Therefore, Examination 1 aims to investigate the neck rotation pattern of the low score group in a left large space, and Examination 2 aims to determine the neck rotation pattern of the group that neglects the leftmost end area in a large space. In this study, based on the data obtained in our previous study, we set angular bands in increments of 5 degrees to the left and right from the midline, calculated the total search time in each angular band, and analyzed them in detail.

Understanding the visual search pattern of patients with USN performing a continuous task in a large space is important for the development of an effective rehabilitation program for left-sided exploration. The present study aimed to understand the symptoms of USN, and develop more effective rehabilitation 
program. The details of the participants, methods, experimental settings, and a figure are the same as in our previous report [7].

\section{Methods}

\subsection{Participants}

This study included nine patients with left USN due to right hemisphere damage caused by cerebrovascular disease (USN group, mean age $71.9 \pm 12.2$ years, Table 1), nine patients with right hemisphere damage but without USN (non-USN group, mean age $68.9 \pm 12.0$ years), and six healthy individuals with no history of brain damage (healthy adult group, mean age $69.0 \pm 2.1$ years) who were age-matched to the right hemisphere damage groups. The USN and non-USN groups were admitted to a research collaborative hospital (Mie, Japan) between 2009 and 2011. Patients fulfilled the criteria and conditions of this study and provided full consent to participate in this research. Causes of USN in the non-USN group were infarction and hemorrhage. Brain lesions in the USN and non-USN groups were characterized using CT scans and MRI. Brain lesion locations of the non-USN group included the putamen, frontal and temporal lobes, and thalamus. The absence of USN in the healthy adult group was confirmed using line bisection and cancellation tests. Those with eye movement paralysis or visual field defects were excluded from the study. A Mini Mental State Examination (MMSE) was performed to exclude individuals with disturbance of consciousness, dementia, and severe attention deficits, and only subjects who scored above the cut-off point were included in the study. The MMSE is an intelligence evaluation scale that includes orientation, memory, and calculation [8]. The maximum score is 30 points, whereby 24 points or more is regarded as healthy, and 23 points or less is regarded as abnormal. The MMSE average score of the non-USN group was $27.6 \pm 2.5$ points.

Table 1. Demographic and clinical characteristics of patients with USN.

\begin{tabular}{cccccccc}
\hline $\begin{array}{c}\text { Patient } \\
\text { No. }\end{array}$ & Sex & $\begin{array}{c}\text { Age } \\
\text { (years) }\end{array}$ & $\begin{array}{c}\text { Cause of } \\
\text { USN }\end{array}$ & Lesion location & $\begin{array}{c}\text { Days } \\
\text { since } \\
\text { onset }\end{array}$ & Hemianopia & $\begin{array}{c}\text { BITC } \\
\text { score }\end{array}$ \\
\hline 1 & F & 79 & Infarction & Parieto-occipital & 37 & No & 35 \\
2 & M & 59 & Hemorrhage & Putamen & 135 & No & 100 \\
3 & M & 73 & Infarction & Frontal & 164 & No & 130 \\
4 & F & 85 & Hemorrhage & Thalamus & 70 & No & 49 \\
5 & M & 78 & Hemorrhage & Temporo-occipital & 106 & No & 111 \\
6 & M & 80 & Aneurysm & Temporal & 161 & No & 114 \\
7 & M & 46 & Hemorrhage & Putamen & 56 & No & 117 \\
8 & F & 70 & Hemorrhage & Putamen & 173 & No & 128 \\
9 & F & 77 & Infarction & Parietal & 126 & No & 123 \\
\hline
\end{tabular}

BITC $=$ Behavioural Inattention Test Conventional subtest (Japanese edition), maximum score $=146$. 
All subjects with right hemisphere damage underwent a desk-based Behavioural Inattention Test Conventional Subtest (BITC, Japanese edition). In clinical practice, the BITC is commonly used to determine the presence and severity of USN in patients with right hemisphere damage. BITC consists of six subtests, and its maximum total score is 146 points. The cut-off point is 131 points, and if the total score is less than that, the patient is considered to exhibit symptoms of USN [9]. In this study, the subjects with right hemisphere damage who scored below the cut-off point were included in the USN group, and those scoring above the cut-off were included in the non-USN group. The BITC average score of the non-USN group was $143.2 \pm 4.0$ points.

This study was approved by the Ethics Committee of Kanazawa University. Participants were given a complete explanation of the study, and only those who provided informed consent were included.

\subsection{Visual Search Test}

The subject's back and shoulders were placed against the backrest of the chair, which was positioned parallel to the screen. The chair was placed only $1.5 \mathrm{~m}$ from the vertical center of the screen $(170 \mathrm{~cm}$ high $\times 240 \mathrm{~cm}$ wide), so that head turning and/or eye movements were necessary to accomplish the visual search task; additionally, the task was designed so that it was difficult for the subject to use eye movements alone to perform the visual search (Figure 1). The subject observed a red circle at the center of the screen; this was considered to be the initial head position.

The subjects were tested for horizontal visual search ability over a large field. Before starting the visual search test, subjects were shown the shapes used in the task and instructed to identify and speak out the name of each shape to ensure that there were no disabilities in speech, vision, and cognition. They were

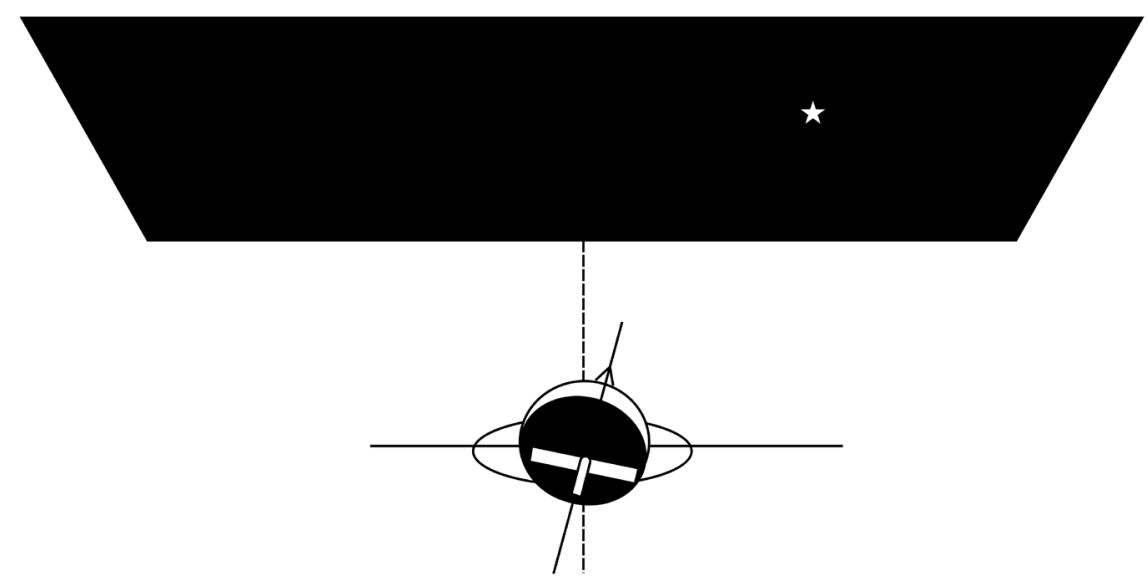

Figure 1. An aerial view diagram of the visual search task in a large space for patients with unilateral spatial neglect and controls. The visual target is represented as the star on the screen. The patient is represented as facing the screen with the axis of neck turning drawn through the nose and the back of the head. (Adapted from Nakatani K., et al. 2013). 
allowed to visually explore the field and were instructed to identify the observed shapes as quickly as possible. The observer recorded responses, determined right and wrong answers, and calculated the total number of tasks accomplished.

Visual stimuli comprised shapes that were horizontally displayed in random order (Takara, Matsusaka, Mie, Japan). The target shapes used for the task were easily identifiable and simple shapes such as circles, triangles, and cubes. Each set consisted of a continuous display of 18 shapes, where each shape was displayed for 3.5 seconds. The time taken to display each set was approximately 1 minute, 10 seconds.

The screen was bisected vertically into two halves (left and right) and three vertical zones were created on each side, yielding a total of six zones. The zones from the far left of the field to the center were named left 3 (L3), left 2 (L2), and left 1 (L1). The zones from the far right of the field to the center were named right 3 (R3), right 2 (R2), and right 1 (R1). These six zones were assigned only for the purpose of obtaining data and were not displayed on the screen. During the interval between tasks, a red circle was displayed at the center of the screen. The room was dimly illuminated. An ultra-short focus model LCD projector (XP-50, Sanyo, Osaka, Japan) was used to project the sets onto the screen.

\subsection{Measuring Cervical Rotation Angle during the Visual Search Test}

Cervical rotation angle was measured using a rotary potentiometer (DI-Angle Rotator, Hikari Bellcom, Kanagawa, Japan). By using an electronic tool to measure the joint angle, we were able to continuously measure the rotation angle throughout the task, even during movement. The DI-Angle Rotator is composed of an angle gauge, a digital display device, and an analog-to-digital converter. The tool was equipped with a built-in light angle sensor, and a movable arm of the elastic body that allowed it to adapt to various movements. The movable arm was fixed to the occiput using a flexible band. Subjects could move their heads freely. The arm weighs only $120 \mathrm{~g}$, and subjects in a preliminary experiment reported no discomfort during movement while using the device. The perceived angle in the horizontal plane was recorded every 20 milliseconds. Cervical rotation angle data were processed using Microsoft Excel (Microsoft, Seattle, WA, USA).

To record and indicate the neck rotation angle during the visual search task in a large space, the angular bands were set in increments of 5 degrees to the left and right of the midline. The cumulative total search time in each angular band was calculated from the record of the neck rotation angle during the visual search task.

Statistical analysis was carried out using the Statistical Package for the Social Sciences Version 24 (IBM, Chicago, IL, USA). The $t$ test and Pearson's correlation coefficient study were performed, and the level of significance was set at $\mathrm{p}<$ 0.05 . The $t$ test was analyzed after confirming homoscedasticity by $\mathrm{F}$ test. 


\section{Results}

\subsection{Relationship between Neglect in a Large Space on the Left Side and Neck Rotation Pattern}

In Examination 1, we investigated the neck rotation pattern of the low score group, during the visual search task in a large space (Figure 2). The non-USN group with right hemisphere damage and the healthy adult group yielded a perfect score of 36 points in this task. The 18 patients with right hemisphere damage were divided into two groups, $\geq 18$-point group $(n=9)$ and $<18$-point group $(n=9)$, based on the total score of the L3-L1 areas (Table 2). This group classification was consistent with the group composition "with and without USN" based on the BITC cut-off score. The average BITC score was 143.2 points in the $\geq 18$-point group and 100.8 points in the $<18$-point group. A strong correlation was observed between the total score of L3-L1 areas of the visual search task and that of BITC $(r=0.75, p<0.01)$. A comparison of the average value of the search time for each angular band between the two groups revealed that the total value of the search time of the $<18$-point group was significantly large in the angular band of 5 - 9 degrees to the right $(\mathrm{t}=2.52, \mathrm{df}=16, \mathrm{p}<0.05)$. When the angular band of 5 - 9 degrees to the right was excluded, the total value of the search time of the $<18$-point group was significantly smaller $(\mathrm{t}=2.40, \mathrm{df}=16, \mathrm{p}<0.05)$. Furthermore, the total value of the search time to the right of the $<18$-point group was significantly larger when the median 0 degree was excluded $(t=2.42$, $\mathrm{df}=16, \mathrm{p}<0.05)$. No significant difference was observed to the left after excluding the median 0 degree.

In clinical practice, therapists instruct patients with USN to "Rotate your neck widely to find the targets" in order to increase their attention toward the

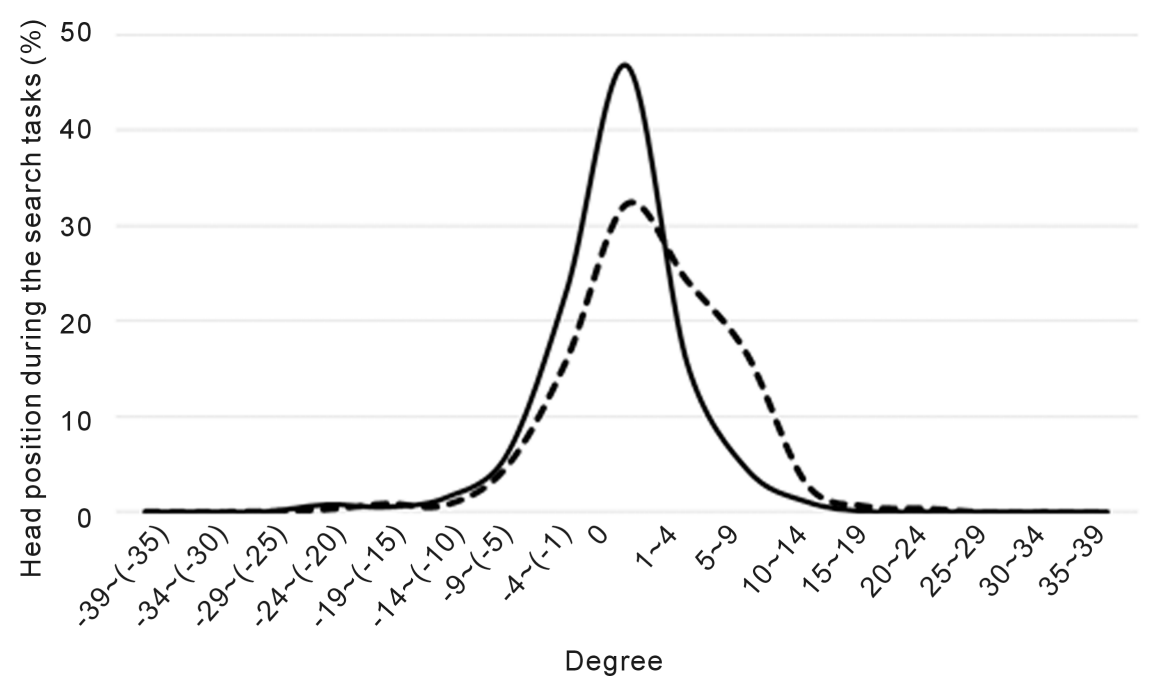

Figure 2. Head position during the visual search task in a large space (\%). Rotation patterns in subjects in the $<18$-point group (dotted line) were compared with those in subjects in the $\geq 18$-point group (solid line). Rotations, grouped into $5^{\circ}$ increments, are shown on the $\mathrm{x}$-axis, with $0^{\circ}$ rotation at the center of the screen. The head position over the entire time was considered $100 \%$. 
Table 2. Scores for each area in the visual search task in a large space: Focus on the total score of L3-L1.

\begin{tabular}{cccccccc}
\hline & Total & L3 & L2 & L1 & R1 & R2 & R3 \\
\hline the <18-point group & 27.4 & 1.7 & 3.2 & 4.8 & 5.8 & 6 & 6 \\
the 218 -point group & 36 & 6 & 6 & 6 & 6 & 6 & 6 \\
Healthy group & 36 & 6 & 6 & 6 & 6 & 6 & 6 \\
\hline
\end{tabular}

$\mathrm{L}=$ left, $\mathrm{R}=$ right. The maximum score for each area is 6 , maximum total score of $\mathrm{L} 3-\mathrm{L} 1$ is 18 , and maximum total score of this task is 36 . According to the score on the left space (L3-L1), patients with right hemisphere damage were classified into two groups.

neglected side. Therefore, we multiplied the neck rotation angle (absolute value from the midline) with the search time at the angle and graphed it with the median value of each angular band (Figure 3). This method analyzes neck rotation and its duration with meaning as "search effort" and "compensation strategies". Simultaneously, we detected the stopping or continuation of the search in the right space and the "rapid search movement during the search task", which is occasionally found in USN patients. Because we have already reported in our previous study that the head position stays in the midline for the longest time, the midline 0 degree was excluded (Figure 4).

\subsection{Relationship between the Score of the Leftmost End Area (L3) and Neck Rotation Pattern}

USN patients tend to focus on the right side. The perception of targets in a space and the reaction to them are processed first from the space on the right. In the case of USN, the neglect symptom increases toward the left visual space, particularly toward the left end, and this tendency is more significant in severe USN patients. Therefore, in Examination 2, the neck rotation pattern was examined by focusing on its relationship with the score of the leftmost end area (L3) in a large space (Figure 4). Based on the score of L3 (perfect score: 6 points), a score of $\geq 5$ points $(n=11)$ was classified as a high score group in L3 and that of $<5$ points $(n=7)$ was classified as a low score group in L3 (Table 3$)$. The average score of the high score group was $5.82 \pm 0.4$ and that of the low score group was $0.71 \pm 1.25$. A strong correlation was observed between the score of L3 and the total score of BITC $(r=0.72, p<0.01)$. The average values of the search time were compared for each angular band in increments of 5 degrees among these two groups. We observed that the search time of the low score group in L3 was significantly smaller in the angular band of $1-4$ degrees to the left $(t=2.15, \mathrm{df}=$ $16, p<.05)$. When the angular band of $1-4$ degrees to the left was excluded, the total value of the search time of the low score group in L3 was significantly larger $(t=2.13, d f=16, p<0.05)$. In the angular band of $5-9$ degrees to the right, the total value of the search time of the low score group in L3 tended to be larger $(\mathrm{t}$ $=1.92, \mathrm{df}=16, \mathrm{p}=0.072$ ). The total value of the search time to the right of the low score group was significantly larger when the median 0 degree was excluded $(t=2.35, d f=16, p<0.05)$. No significant difference was observed to the left 


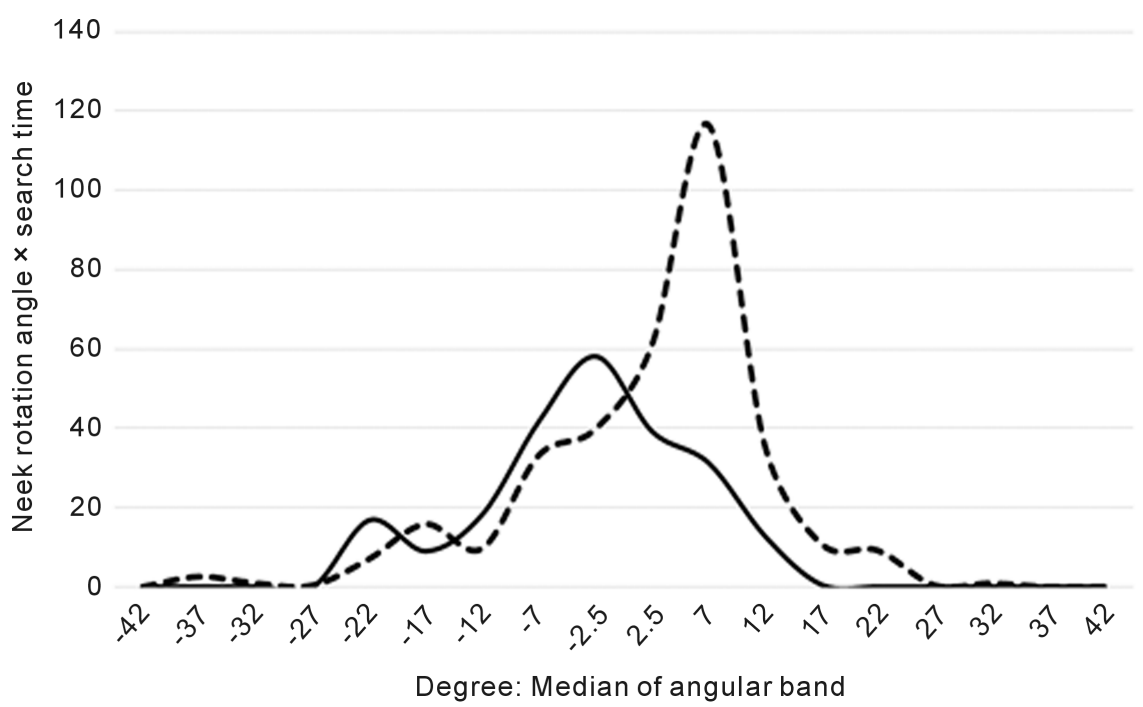

Figure 3. Rotation patterns in subjects in the $<18$-point group (dotted line) were compared with those in subjects in the $\geq 18$-point group (solid line). The midline 0 degree is excluded.

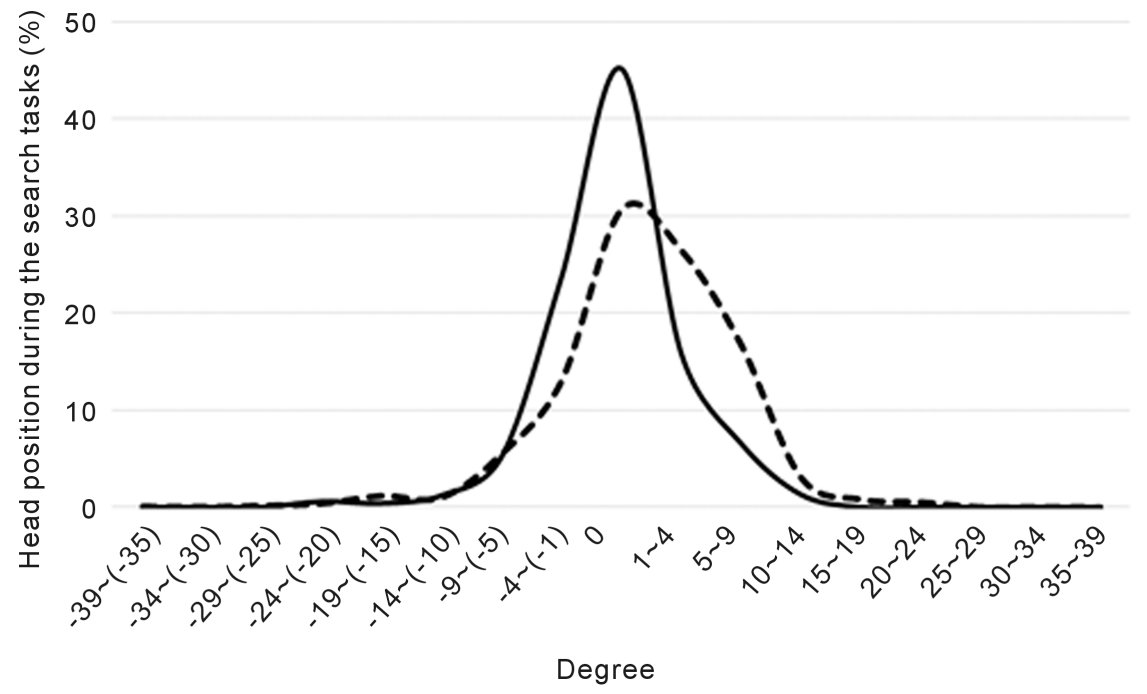

Figure 4. Head position during the visual search task in a large space (\%). Rotation patterns in subjects in the low score group (dotted line) were compared with those in subjects in the high score group (solid line). Rotations, grouped into $5^{\circ}$ increments, are shown on the $\mathrm{x}$-axis, with $0^{\circ}$ rotation at the center of the screen. The head position over the entire time was considered $100 \%$.

Table 3. Scores for each area in the visual search task in a large space: Focus on the score of L3.

\begin{tabular}{cccccccc}
\hline & Total & L3 & L2 & L1 & R1 & R2 & R3 \\
\hline low score group in L3 & 27.4 & 0.7 & 2.4 & 4.4 & 5.7 & 6 & 6 \\
high score group in L3 & 35.8 & 5.8 & 6 & 6 & 6 & 6 & 6 \\
\hline
\end{tabular}

$\mathrm{L}=$ left, $\mathrm{R}=$ right. The maximum score for each area is 6 , and maximum total score of this task is 36 . According to the score of $\mathrm{L} 3$, patients with right hemisphere damage were classified into two groups. 
after excluding the 0 degree. The average BITC score was 140.0 points in the high score group and 93.7 points in the low score group.

Using the aforementioned method, we multiplied the neck rotation angle (absolute value from the midline) with the search time at the angle, and graphed it as the median value of each angular band (Figure 5).

\section{Discussion}

In a previous study, we reported a strong correlation between the number of visual search tasks accomplished in a large space and the total BITC score, and no significant difference was observed in the neck rotation angle as well as the average and maximum values of the group without USN including normal healthy adults and individuals with USN, under experimental settings [7]. In addition, the neck rotation pattern of the group with USN presented a tendency to search in the right space [7]. In this experimental setting, the non-USN group with right hemisphere damage and healthy adult group obtained the highest score (36 points) in the visual search task in a large space. This group classification was consistent with the group composition "with and without USN" based on the BITC cut-off score. Therefore, the search task in a large space used in this study and analyses based on that score are considered appropriate for examining the symptoms of USN. Even in patients with dementia, if the MMSE score is at least 15 points, the test and examination of USN can be conducted [10]. In this study, only subjects who scored more than the cut-off point were included. Therefore, the possibility that disturbances of consciousness, severe dementia, and/or lack of comprehension have influenced the present findings is extremely low. Based on these factors, we discuss the results and findings obtained in this study.

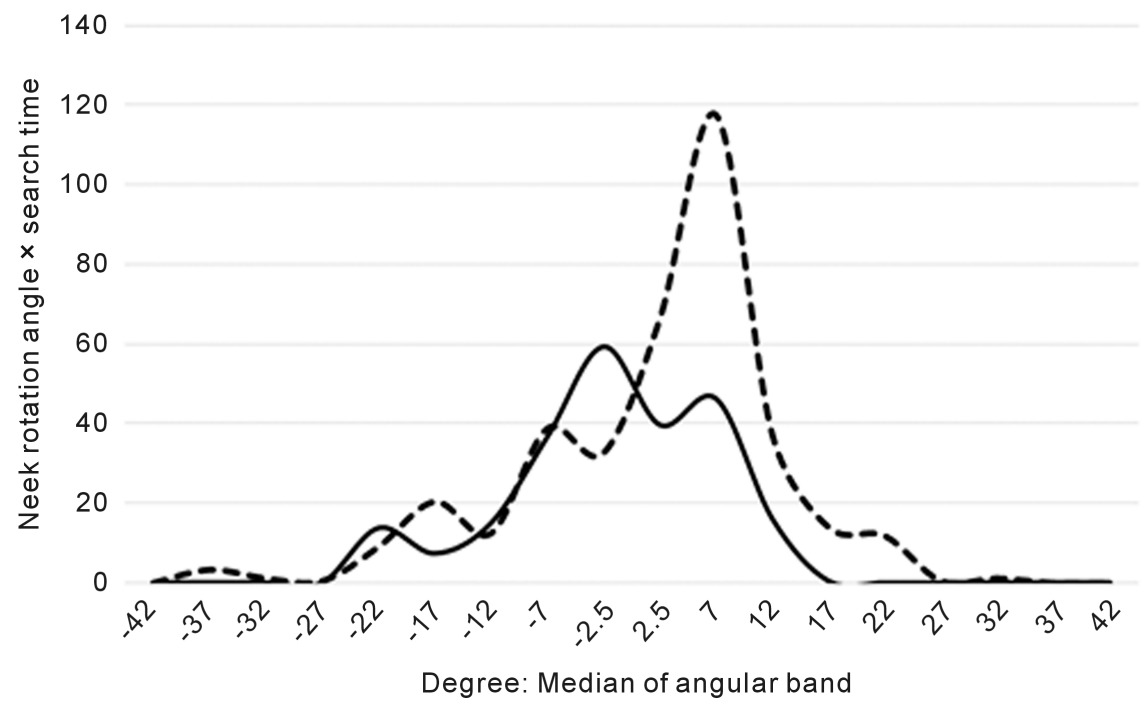

Figure 5. Rotation patterns in subjects in the low score group (dotted line) were compared with those in subjects in the high score group (solid line). The midline 0 degree is excluded. 
In Examination 1, the 18 patients with right hemisphere damage were divided into the $\geq 18$-point and $<18$-point groups, based on the total score of the L3-L1 areas. We subsequently investigated the neck rotation pattern of the two groups during the visual search task in a large space (Figure 2, Figure 3). The results revealed that the total value of the search time of the $<18$-point group was significantly larger in the angular band of 5 - 9 degrees to the right. In the same group, which was corresponding to the USN group, the search time was longer in the angular bands of 5 - 9 on the right, compared with the non-USN group. This may explain why the neck rotation pattern of the USN group indicated a bell-shaped distribution slightly to the right. It is also suggested that the frequency of rotating the head to other angular bands is low.

In Examination 2, the neck rotation pattern was examined by focusing on its relationship with the score of the leftmost end area (L3) in a large space (Figure 4 , Figure 5). The search time of the low score group in L3 was significantly small in the angular band of 1 - 4 degrees to the left. The total value of the search time to the right of the low score group was significantly larger after excluding the median 0 degree. This suggests a high frequency of rotating the head to other angular bands. In the angular band of 5 - 9 degrees to the right, the total value of the search time of the low score group in L3 tended to be large. USN patients tend to focus on the right side. The tendency of neglect symptoms to increase particularly toward the left end is more significant in severe USN patients. The total value of the search time of the low score group in L3 was significantly smaller in the angular band of left 1 - 4 degrees, and there was a tendency to focus at the angular band of right 5 - 9. This creates the impression that USN patients face "somewhat to the right". In the experimental settings of this study, it was observed that this impression was not derived from the extreme right side, but in the angular band slightly to the right of the midline.

In clinical settings, although USN patients are instructed to rotate their heads to the neglected side, it is seldom reported that these patients focus on the left side spontaneously. Therefore, as the first realistic goal, therapists instruct UNS patients to divert their focus to the left side next to the midline, but it is difficult for many patients to even cross the midline. A significant decrease in the search time in the left 1 - 4 degrees angular band may provide the cue to interpret the difficulty in crossing the midline to the left for USN patients.

An angular band of 5 - 9 degrees to the right and that of 1 - 4 degrees to the left represent very narrow areas around the midline. The significance of focusing on them is prism adaptation using prism eyeglasses, where the field of view appears shifted 10 or 15 degrees to the right; it is one of the rehabilitation strategies for USN [11]. As an "after effect" of the removal of prism eyeglasses, there is a method to confirm that the action finger pointing to the target is slightly shifted to the left [12]. The aforementioned angular bands focused on in this study are almost the same as the angle of shift of prism adaptation and that of the after effect. Although it is a small angular range of approximately 10 degrees to the left 
and right from the median, focusing on these angular bands seems critical to understand USN symptoms.

Regardless of USN, the head of the subjects was mostly in the center, and the center of the search pattern was at the midline. The subjects could maintain their posture in the midline possibly because of equilibrium sensation. In brief, equilibrium sensation is determined by multiple stimuli, such as information from the vestibular organs because of changes in the head position and information on changes in neck rotation or that on joint position sensation because of the posture during the experiment. USN patients tend to focus on the right side. The perception of targets in a space and the reaction to them are processed first from the space on the right. The visual search pattern in the USN group was shifted toward the ipsilateral side for the entire frame [13]. There was a shift to the right in subjects with USN who reported looking straight [14] [15]. The present findings, regarding the visual search pattern of USN patients in the angular band of 5 - 9 degrees to the right and that of 1 - 4 degrees to the left also indicated that the visual search pattern and neck position during the search task of USN patients was slightly shifted to the right side.

Setting the subjective midline at the midline or shifting it to the neglected side is suggested to improve left-sided visual perception in USN patients. For correcting the rightward deviation, there is a method of turning the trunk to the left [16]. Neck vibration has been suggested as a method for shifting the subjective midline toward the neglected side. Vibration of the contralateral neck muscle is suggested to lead to a temporary shift of the subjective midline toward the contralesional hemispace. In addition, it is believed to improve neglect symptoms [17] [18] [19] [20]. However, generalization is difficult in USN patients. It remains to be investigated whether these stimuli or neck vibration result in changes in the neck rotation pattern.

\section{Conclusion}

Because therapists instruct USN patients to rotate their heads to the neglected side, we examined the neck rotation pattern of these patients at search tasks in a large space. However, it is clear that gaze plays an important role in the ability to accurately capture the target. In order to investigate the visual search pattern, verification with the eye mark recorder camera is considered necessary, and we are currently conducting studies using such an approach. The diversity of neglect symptoms and effectiveness of neck rotation of USN patients have not yet been completely elucidated. It may be difficult to apply the present findings directly to daily life situations. However, we believe that the present findings, which were obtained using quantitative data of the cervical rotation pattern, are significant. We believe that our results may be used to promote further understanding of USN symptoms, reduce collision risk by oversight, and develop effective training methods. We hope that the results of this study will improve the understanding of USN and, subsequently, aid the development of effective rehabilitation programs. 


\section{Acknowledgements}

This research was supported by JSPS KAKENHI Grant Number 26350919 (research leader: Ken Nakatani).

\section{References}

[1] Heilman, K.M., Watson, R.T. and Valenstein, E. (1985) Neglect and Related Disorders. In: Heilman, K.M., et al., Eds., Clinical Neuropsychology, 2nd Edition, Oxford University Press, New York, 243-293.

[2] Rapcsak, S.Z., Cimino, C.R. and Heilman, K.M. (1988) Altitudinal Neglect. Neurology, 38, 277-281. https://doi.org/10.1212/WNL.38.2.277

[3] Shelton, P.A., Bowers, D. and Heilman, K.M. (1990) Peripersonal and Vertical Neglect. Brain, 113, 191-205. https://doi.org/10.1093/brain/113.1.191

[4] Halligan, P.W. and Marshall, J.C. (1991) Left Neglect for Near but Not Far Space in Man. Nature, 350, 498-500. https://doi.org/10.1038/350498a0

[5] Mennemeier, M., Wertman, E. and Heilman, K.M. (1992) Neglect of Near Peripersonal Space. Evidence for Multidirectional Attentional Systems in Humans. Brain, 115, 37-50. https://doi.org/10.1093/brain/115.1.37

[6] Sunahara, N. and Notoya, M. (2005) Relationship between Sound Lateralization Abilities and Laterality Index by Behavioural Inattention Test in Unilateral Spatial Neglect Patients. Journal of the Tsuruma Health Science Society, 29, 11-19.

[7] Nakatani, K., Notoya, M., Sunahara, N., et al. (2013) Horizontal Visual Search in a Large Field by Patients with Unilateral Spatial Neglect. Journal of Clinical Neuroscience, 20, 837-841. https://doi.org/10.1016/j.jocn.2012.07.014

[8] Folstein, M.F., Folstein, S.E. and McHugh, P.R. (1975) Mini-Mental State. A Practical Method for Grading the Cognitive State of Patients for the Clinician. Journal of Psychiatric Research, 12, 189-898. https://doi.org/10.1016/0022-3956(75)90026-6

[9] Ishiai, S. (1999) Behavioural Inattention Test. Japanese Edition, Shinkoh-Igaku Publisher, Tokyo.

[10] Ishiai, S., Koyama, Y., Seki, K., et al. (2000) Unilateral Spatial Neglect in AD: Significance of Line Bisection Performance. Neurology, 8, 364-370.

https://doi.org/10.1212/WNL.55.3.364

[11] Rossetti, Y., Rode, G., Pisella, L., et al. (1998) Prism Adaptation to a Rightward Optical Deviation Rehabilitates Left Hemispatial Neglect. Nature, 395, 166-169. https://doi.org/10.1038/25988

[12] Frassinetti, F., Angeli, V., Meneghello, F., et al. (2002) Long-Lasting Amelioration of Visuospatial Neglect by Prism Adaptation. Brain, 125, 608-623. https://doi.org/10.1093/brain/awf056

[13] Karnath, H.O., Niemeier, M. and Dichgans, J. (1998) Space Exploration in Neglect. Brain, 121, 2357-2367. https://doi.org/10.1093/brain/121.12.2357

[14] Karnath, H.O. (1997) Spatial Orientation and the Representation of Space with Parietal Lobe Lesions. Philosophical Transactions of the Royal Society B: Biological Sciences, 352, 1411-1419. https://doi.org/10.1098/rstb.1997.0127

[15] Ferber, S. and Karnath, H.O. (1999) Parietal and Occipital Lobe Contributions to Perception of Straight Ahead Orientation. Journal of Neurology, Neurosurgery, and Psychiatry, 67, 572-578. https://doi.org/10.1136/jnnp.67.5.572

[16] Karnath, H.O., Schenkel, P. and Fischer, B. (1991) Trunk Orientation as the Determining Factor of the Contralateral Deficit in the Neglect Syndrome and as the 
Physical Anchor of the Internal Representation of Body Orientation in Space. Brain, 114, 1997-2014. https://doi.org/10.1093/brain/114.4.1997

[17] Karnath, H.O., Christ, K. and Hartje, W. (1993) Decrease of Contralateral Neglect by Neck Muscle Vibration and Spatial Orientation of Trunk Midline. Brain, 116, 383-396. https://doi.org/10.1093/brain/116.2.383

[18] Karnath, H.O. (1995) Transcutaneous Electrical Stimulation and Vibration of Neck Muscles in Neglect. Experimental Brain Research, 105, 321-324. https://doi.org/10.1007/BF00240969

[19] Schindler, I., Kerkhoff, G., Karnath, H.O., et al. (2002) Neck Muscle Vibration Induces Lasting Recovery in Spatial Neglect. Journal of Neurology, Neurosurgery, and Psychiatry, 73, 412-419. https://doi.org/10.1136/jnnp.73.4.412

[20] Johannsen, L., Ackermann, H. and Karnath, H.O. (2003) Lasting Amelioration of Spatial Neglect by Treatment with Neck Muscle Vibration Even without Concurrent Training. Journal of Rehabilitation Medicine, 35, 249-253.

https://doi.org/10.1080/16501970310009972 